\title{
A pilot study of the primary care management of knee osteoarthritis in the Northern States of Malaysia.
}

\author{
Arshad A and Rashid R \\ Putra Specialist Centre, Alor Setar, Kedah, Malaysia \\ ABSTRACT
}

\begin{abstract}
Introduction: Primary care management of knee osteoarthritis $O A$ has received little attention in the scientific literature and the main reason of this survey is to study and explore the variations and patterns of primary care management and assess both conventional and complementary therapy usage in knee OA in the primary care setting. Materials and Methods: A cross sectional survey of 100 randomly selected general practitioners (GPs) in the northern states of Malaysia (Kedah, Perlis, Pulau Pinang) was undertaken using questionnaires. The GPs involved were asked about basic knowledge of OA in terms of diagnosis, investigation, and treatment of OA. They were also asked their usage of conventional and complementary medication. Results: 80 (80\%) GPs responded to the questionnaires sent. $85 \%$ of GPs were in solo practice and $15 \%$ in group practice. Most of the GPs surveyed (69\%) were in practice for more than 10 years, $21 \%$ in $5-10$ years and $10 \%$ were in practice for less than 5 years. $65 \%$ GPs surveyed see an average of more than 20 patients per week, $25 \%$ see about 10 20 patients and $10 \%$ see less than 10 patients per week. $75 \%$ of GPs surveyed would arrange an X-ray. $65 \%$ of GPs surveyed will arrange a blood test, mostly serum uric acid, rheumatoid factor and ESR. Pharmacological management consists of first line treatment with analgesics (32\%), NSAIDs (59\%) or a combination of the two (4\%). Non-pharmacological management consist of advise an exercise (37\%), weight reduction (23\%) and referral to physiotherapy (8\%). $89 \%$ of GPs surveyed prescribed some form of complementary medications. $68 \%$ prescribed glucosamine sulphate, $29 \%$ chondroitin sulphate, $18 \%$ cod liver oil, $12 \%$ evening primrose oil. Only 5\% of GPs surveyed perform intra- articular injection. Conclusion: The data suggest that in the primary care, majority of GP over investigate the diagnosis of OA. Pharmacological interventions largely concentrate on analgesic and NSAIDs. The use of physiotherapy and non drug approach were enormously under-utilized. There is a need to further educate GPs in the management of OA.
\end{abstract}

KEYWORDS: Osteoarthritis, Management, Primary care

\section{INTRODUCTION}

Musculoskeletal disorders represent a large proportion of consultations in primary care, making up some $18 \%$ of the workload of general practitioners (GPs). ${ }^{1}$ Osteoarthritis (OA) is the commonest disorders of all rheumatic diseases and is almost a universal problem in people aged over 65 year, however, it is more uncommon in people aged less than $45 . .^{2,3} \mathrm{OA}$ is more common among men than women under age of 45 and more common among women than men over age of 54. Clinically, patterns of joint involvement also demonstrate sex differences with women having average more joints involvement and more frequent complaints of joint stiffness and joint swelling. ${ }^{4}$ It is a progressive disease characterized by the degeneration of articular cartilage. Previously considered as purely a degenerative disease that was an inevitable

Corresponding Author:

Dr. Anwar Arshad,

Consultant Rheumatologist Putra Specialist Center

Alor Star, Kedah, Malaysia

Email: anwararshad@hotmail.com consequence of aging and trauma, $\mathrm{OA}$ is now viewed as metabolically dynamic, essentially reparative process that is increasingly amenable to treatment. ${ }^{5}$ $\mathrm{OA}$ is also a single most important cause of loco-motor disability and a major challenge to health care and is perceived as an extremely costly disease in our society not only in terms of direct cost to health care systems, but also in terms of indirect costs attributable to lost work days, reduced production, invalidity payments etc. ${ }^{6}$

Management of OA requires careful diagnostic evaluation, appreciation of the severity of the articular process, establishment of the level of necessary or desired therapy and a judgement on general health status before initiating any type of intervention. The main objectives in the management of $\mathrm{OA}$ are to reduce symptoms, minimize functional disability and limit progression. Current modern management of OA involves a wide variety of health professionals. ${ }^{7,8}$ Given the number of professions involved in treatment and the importance of service provision for the successful management of chronic conditions, it is perhaps surprising that there is very limited evidence on how different groups view the management of OA especially in the primary care setting. ${ }^{9.12}$ Most of the 
specialist society or organizations, who has major interest in OA such as rheumatology, has developed treatment guidelines for $\mathrm{OA} .{ }^{13,14}$ In our region, majority of those suffering from OA will first see their GPs. The primary care management of $\mathrm{OA}$, to date, has received little attention in the scientific literature and little is known on the management of OA by GPs in the community. The main reason of this survey is to study and explore the variations and patterns of primary care management and to assess the usage of both conventional and complementary therapy in knee OA in the primary care setting. The data and information obtained from this study will identify weaknesses and help to improve healthcare system in managing this common disease in the primary care setting.

\section{MATERIALS AND METHODS}

A cross sectional survey of 200 randomly selected general practitioners (GPs) in the Northern States of Malaysia which comprise of 3 states (Kedah, Perlis, Penang) was undertaken using a standard postal questionnaire. The GPs names and addresses of their practices were retrieved from the' Private Medical Practitioners Society ' (PMPS) data base of the respective states. The questionnaires were mailed to the selected GPs. The GPs involved were asked about basic knowledge of OA in terms of diagnosis, investigation, and treatment pattern of OA. They were also asked about the usage of conventional and complementary treatment. A reminder was sent to all selected GPs about a month after the first mail were sent out. All data were analysed after the end of the third month.

\section{RESULTS}

A total of 80 (80\%) GPs completed and responded to the 3-page questionnaires that addresses various aspects of OA and its treatment. $68(85 \%)$ of GPs were in solo practice and $12(15 \%)$ in group practice. Most of the GPs surveyed $55(69 \%)$ were in practice for more than 10 years, $15(21 \%)$ in 5 - 10 years and $8(10 \%)$ were in practice for less than 5 years. 52 (65\%) GPs surveyed see an average of more than 20 OA patients per week, 20(25\%) see about 10- 20 patients and $18(10 \%)$ see less than 10 patients per week. 60 (75\%) of GPs surveyed would arrange for a radiograph to be taken, for various reasons including: diagnosis 60 (70\%), to utilize their own X-ray machine $4(6 \%)$, at patient's request 16 (20\%). 52 (65\%) of GPs surveyed will arrange a blood test for diagnostic purposes, mostly serum uric acid, rheumatoid factor and ESR. When asked about which agent would be their first choice of treatment; non steroidal anti-inflammatory drugs (NSAIDs) was ranked first among $48(59 \%)$ of GPs, followed by analgesics 63 (35\%) and surprisingly not many GPs 3 (4\%) would combine those 2 agents. Non- pharmacological management were relatively under-utilised by most of the GPs who took part in this study as only 30 (37\%) GPs advise exercise and 18 (23\%) would advise for weight reduction while referrals to physiotherapy were only $6(8 \%)$. Most of the GPs 71 (89\%) surveyed prescribed some form of complementary medications,
$54(68 \%)$ of GPs prescribed glucosamine, followed by 23 (29\%) chondroitin, 14 (18\%) cod liver oil, and 9 (12\%) evening primrose oil. Only 4 (5\%) of GPs surveyed perform intra- articular injection. Majority of the GPs 76 (95\%) are not aware of the existence of the National guidelines of the management of OA.

\section{DISCUSSION}

This study describes the views of GPs treating OA in a primary care setting. The management of OA should involve a multidisciplinary approach with the aim to relieve symptoms and improve joint function. However, this study proves that GPs were less likely to use or to refer OA patients to the appropriate support groups or to utilize non-pharmacological agents but are more likely to use complementary therapy. GPs were also less likely to consider minor surgical procedures such as intra-articular injection.

Our findings are consistent with Chard et al where they noted that GPs in Britain used complementary and alternative medicine more than rheumatologists, although our study did not make such comparison, it still demonstrated the same pattern. ${ }^{15}$ There are several possible explanations for this. First, there may be differences in attitude to treatment as those practiced in primary care may be more willing to accept the 'non-scientific' complementary approaches. Secondly, GPs may be more responsive to patient's preferences than following guidelines which is evidence based. Fewer regards to support groups and non pharmaceutical approach by GPs are the two important issues found in our study, as it may reflect lack of knowledge of support groups by doctors in the primary care setting. The lower proportion of GPs who would chose to use intra-articular injection as a treatment option may suggest that GPs are reluctant to refer for this type of treatment, also revealed that knowledge of the holistic approach involving a rheumatologist might still be lacking.

In terms of investigations, clearly majority of GPs over investigate in making the diagnosis of OA. Radiological investigation may be able to establish the diagnosis and severity but not always correlate well with symptoms as patients with abnormal radiographs may be asymptomatic. Blood tests are not necessary when diagnosis is certain, however from this study; more than half of GPs involved ordered a blood test to diagnose $\mathrm{OA}$ and serum uric acid being the most frequently tested for. Patients with typical feature of OA may be erroneously diagnosed as having rheumatoid arthritis(RA), lupus, or gout just because of the rheumatoid factor, anti-nuclear-antibody(ANA) lupus are positive, and high serum uric acid respectively. The prevalence of OA is age related, and likewise rheumatoid factor. ANA and ESR thus certainly confuse the picture.

More needs to be done in terms of education of the GPs by arranging CMEs by focusing on the management of OA. The dissemination of knowledge should be en- 
couraged at all levels, emphasizing the diagnosis and management of OA. Curriculum at the under-graduate levels can be emphasized more on rheumatology. The awareness of 'National Guidelines of Management of $\mathrm{OA}^{`}$ should be increased especially in the primary care setting.

This study is obviously has some limitations. Firstly, there are more than 500 GPs in all the 3 northern states of Malaysia and the results might not represent or reflect the actual views of the whole group and further study with more respondents are therefore needed. Secondly, we did not specify to GPs in our questionnaires the site of OA and its severity as it might cause some confusion to GPs when making the choice of investigations and treatments and finally, demographically, we noted that most GPs participated were those working in rural or remote area therefore they might not have a regular exposure to the various health professionals involves in the management of $\mathrm{OA}$ as those services are largely concentrated in the larger cities.

\section{CONCLUSION}

The data from this study suggest that in the primary care setting, majority of GPs over investigate the diagnosis of OA. Complimentary and alternatives medicine usage were noted to be higher in these group. Non- pharmacological interventions were enormously under- utilized. There is a crying need to further educate GPs in the management of OA.

Nevertheless, this study demonstrates the value of exploring views of the 'front line' groups in relation to a specific condition such as OA. Such studies may become increasingly important as health -care becomes more complicated and demand for more community- based care increases.

\section{REFERENCES}

1. Liddell WG, Carmichael CR, Mc Hugh NJ. Joint and soft tissue injections: a survey of general practitioners. Rheumatology 2005; 44:1043-6

2. Felson DT, Radin EL . What causes knee osteoarthrosis-are different compartments susceptible to different risk- factors? J Rheumatol 2005; 21:181-3

3. McAlindon TE, Cooper C, Kirwan JR, Dieppe PA. Determinants of disability in osteoarthritis of the knee. Ann Rheum Dis 1993; 52: 258-62

4. Reginster JY, ed. Osteoarthritis Clinical and Environmental aspects. New York; SpringerVerlag, 1999.

5. Snaith MY, ed. ABC of Rheumatology. London; BMJ publishing, 1996.

6. Long L, Soeken K, Ernst E. Herbal medicines for the treatment of OA; a systematic review. Rheumatology 2001; 40:77-9

7. Dieppe PA, Chard JA, Faulkner A, Lohmander S. Osteoarthritis. In: Barton S, ed. Clinical
Evidence. London; BMJ Publishing Group, 2001:808-22.

8. Norris P. How 'we' are different from 'them': Occupational boundary maintenance in the treatment of musculo- skeletal problems. Social Health Illness 2001: 23:24-43

9. Hochberg M, Altman RD, Brandt K et al. Guidelines for the medical management of osteoarthritis: Part 11. Osteoarthritis of the knee. Arthritis Rheum 1995; 38:1541-6

10. Tallon D, Chard JA, Dieppe PA . Relation between agendas of the research community and the research consumer. Lancet 2000; 355:2073-40

11. Mazzuca SA, Brandt KD, Katz BP, et al . Comparison of general internist, family physicians, and rheumatologists managing patiemts with symptoms of osteoarthritis of the knee. Arthritis Care Res 1997; 10:289- 99

12. Pots MK, Brandt KD. Various health professions groups beliefs about people with arthritis. J Allied Health 1986; 15:245-56

13. Hochberg MC, Altman RD, Brandt KD et al. ACR guidelines for the medical management of the OA. Arthritis Rheum 1995; 38;1541-6

14. Pendleton A, Arden N, Dougados M et al. EULAR recommendations for the management of knee OA.Ann Rheum Dis 2000; 59; 936-44

15. Chard et al. A comparison of the views of rheumatologists, general practitioners and patients on the treatment of OA. Rheumatology 2000; 41:1208-10 
ABOUT YOUR PRACTICE(Kindly tick an appropriate response)

1. Solo

Group

\begin{tabular}{|c|l|}
\hline yes & \\
\hline no & \\
\hline
\end{tabular}

2. How long you 've been in practice?

\begin{tabular}{|l|l|}
\hline 5 year & \\
\hline $5-10$ year & \\
\hline Above 10 year & \\
\hline
\end{tabular}

\section{ABOUT OSTEOARTHRITIS MANAGEMENT}

3. Average of knee OA seen weekly?

\begin{tabular}{|l|l|}
\hline $\begin{array}{l}\text { Less than } 10 \\
\text { patients }\end{array}$ & \\
\hline $10-20$ patients & \\
\hline $\begin{array}{l}\text { More than } 20 \\
\text { patients }\end{array}$ & \\
\hline
\end{tabular}

4. Do you arrange for x-ray?

\begin{tabular}{|c|l|}
\hline yes & \\
\hline no & \\
\hline
\end{tabular}

If yes, why?

5. Do you do blood test for OA?

If yes, what blood test and why?

\begin{tabular}{|c|c|}
\hline yes & \\
\hline no & \\
\hline
\end{tabular}

If yes, what blood test and why?

6. What drug (or drugs) you commonly prescribed as a first line treatment?

7. Do you recommend any supplements or complimentary medicine?

If yes, what supplement or complementary treatment you prescribe?

\begin{tabular}{|c|c|}
\hline yes & \\
\hline no & \\
\hline
\end{tabular}

8. Do you perform an intra- articular injection?

If yes, which particular joint?

\begin{tabular}{|c|c|}
\hline yes & \\
\hline no & \\
\hline
\end{tabular}


9. Do you recommend non-pharmacological treatment for knee OA?

If yes, what non-pharmacological treatment would you recommend?

10. Do you aware of the clinical practice guidelines on the management of OA produced by The Malaysian Society of Rheumatology?

If yes, do you utilize the guidelines for the management?

\begin{tabular}{|c|l|}
\hline yes & \\
\hline no & \\
\hline
\end{tabular}

\begin{tabular}{|c|c|}
\hline yes & \\
\hline no & \\
\hline
\end{tabular}

THANK YOU FOR YOUR COOPERATION

Corresponding Author: Dr. Anwar Arshad, FACR, MRCP, AM. Consultant Rheumatologist Putra Specialist Center Alor Star, Kedah, Malaysia Tel: +604-734 2888 Fax: +604-7311928

Email: anwararshad@hotmail.com 
